Check for updates

Cite this: Chem. Sci., 2018, 9, 8271

๑ All publication charges for this article have been paid for by the Royal Society of Chemistry

Received 10th July 2018

Accepted 31st August 2018

DOI: $10.1039 / \mathrm{c} 8 \mathrm{sc} 03058 \mathrm{~h}$

rsc.li/chemical-science

\section{Investigation of photocurrents resulting from a living unicellular algae suspension with quinones over time $\uparrow$}

\author{
Guillaume Longatte, ${ }^{\star a}$ Adnan Sayegh, ${ }^{a}$ Jérôme Delacotte, ${ }^{a}$ Fabrice Rappaport, $t^{b}$ \\ Francis-André Wollman, ${ }^{\mathrm{b}}$ Manon Guille-Collignon ${ }^{\mathrm{a}}$ and Frédéric Lemaître (iD *a
}

\begin{abstract}
Plants, algae, and some bacteria convert solar energy into chemical energy by using photosynthesis. In light of the current energy environment, many research strategies try to benefit from photosynthesis in order to generate usable photobioelectricity. Among all the strategies developed for transferring electrons from the photosynthetic chain to an outer collecting electrode, we recently implemented a method on a preparative scale (high surface electrode) based on a Chlamydomonas reinhardtii green algae suspension in the presence of exogenous quinones as redox mediators. While giving rise to an interesting performance $\left(10-60 \mu \mathrm{A} \mathrm{cm}{ }^{-2}\right)$ in the course of one hour, this device appears to cause a slow decrease of the recorded photocurrent. In this paper, we wish to analyze and understand this gradual fall in performance in order to limit this issue in future applications. We thus first show that this kind of degradation could be related to over-irradiation conditions or side-effects of quinones depending on experimental conditions. We therefore built an empirical model involving a kinetic quenching induced by incubation with quinones, which is globally consistent with the experimental data provided by fluorescence measurements achieved after dark incubation of algae in the presence of quinones.
\end{abstract}

\section{Introduction}

Sunlight is the most abundant and sustainable source of energy available on Earth. ${ }^{1}$ That is why strategies have been developed for many years in order to take advantage of solar energy. In this respect, photosynthesis, the biological process evolved by nature to feed the biosphere with reduced carbon, is nowadays a source of inspiration for implementing new and promising conversion strategies. However, despite the very high efficiency of the photochemical converters Photosystems I and II, only a few percent of the total energy available from sunlight is converted into chemical energy. ${ }^{2,3}$ This apparently disappointing performance leads to two opposite considerations. ${ }^{4}$ On the one hand, it could suggest that directly exploiting a very complex system like photosynthesis is not the easiest way to harness solar energy. As a consequence, chemical and electrochemical tools can be used to perform "artificial photosynthesis", i.e. to build photoelectrochemical systems mimicking

${ }^{a}$ PASTEUR, Département de chimie, École Normale Supérieure, PSL University, Sorbonne Université, CNRS, 75005 Paris, France. E-mail: guillaume.longatte@ wanadoo.fr; frederic.lemaitre@ens.fr

${ }^{b}$ Laboratory of Membrane and Molecular Physiology at IBPC, UMR7141 CNRS/ Sorbonne Université, 13 rue Pierre et Marie Curie, 75005 Paris, France

$\dagger$ Electronic supplementary information (ESI) available. See DOI: $10.1039 / \mathrm{c} 8 \mathrm{sc} 03058 \mathrm{~h}$

‡. F. Rappaport passed away before completion of this manuscript. His co-authors wish to dedicate this article to his memory. the basic principles of photosynthesis., ${ }^{\mathbf{5}, \mathbf{6}}$ On the other hand, this lack of efficiency could also suggest that photosynthesis is an unexploited fuel-producing factory. From this point of view, electrochemical tools can be used to harvest electrons from "real" photosynthetic systems (particularly integral and whole systems and under high-light conditions), i.e. to benefit from "natural photosynthesis".

In the case of natural photosynthesis, an outer collecting electrode is used for harvesting the electrons from the given photosynthetic organism. Nevertheless, the implemented strategies and electrochemical configurations strongly depend on the photosynthetic targets. Immobilization of isolated photosynthetic subunits is the most popular way to favour the electrochemical communication between the photosynthetic chain and the electrode. This can be achieved with isolated PSII and PSI, ${ }^{7-13}$ with thylakoid membranes, ${ }^{\mathbf{1 4 - 2 0}}$ or even with isolated chloroplasts. ${ }^{21}$ In many cases, electron relays are often required to enhance the extraction. This is why redox polymers, nanotubes or nanoparticles, and soluble mediators (mainly quinones or $\mathrm{Fe}(\mathrm{CN})_{6}{ }^{3-}$ ) are involved in helping with the electron shuttle from the biological target to the electrode. However, the stability of the system in this approach can be questionable because of the modification of the biological environment and the lack of cell growth. To circumvent this issue, intact biological systems (cyanobacteria, algae...) are also implemented. ${ }^{22-27}$ They can be adsorbed onto the electrode surface or used in suspension. Indeed, a suspension is preferable because it 
potentially ensures the culture and proliferation of the photosynthetic organisms during the photosynthetic electron harvesting. However studies involving cell suspensions on a preparative scale are rather scarce ${ }^{28-34}$ probably because electrical connectivity is made much more difficult due to natural barriers such as membranes or inner compartments that limit direct electron transfer or prevent an exogenous mediator from easily accessing its target.

This is why we recently implemented a set-up involving a suspension of unicellular algae Chlamydomonas reinhardtii on a preparative scale (i.e. conditions where all of the suspension is involved) with a high surface area carbon electrode. ${ }^{35,36}$
Exogenous quinones were used as electron carriers and were selected after comparing and understanding their PSII acceptor behavior as a function of their concentration and illumination conditions. ${ }^{37,38}$ The recorded photocurrents were encouraging in terms of magnitude (10-60 $\mu \mathrm{A} \mathrm{cm}^{-2}$ ) and duration (30 min). However, even though experimental dependences suggested a process that, as a whole, is controlled by the photosynthetic electron extraction, the photocurrents were lower than anticipated, ${ }^{35}$ and not fully consistent with the expected electrocatalytic mechanism involving Michaelis-Menten kinetics. ${ }^{39}$ This suggests that concomitant phenomena occurred during the extraction and contributed to progressively alter the system.

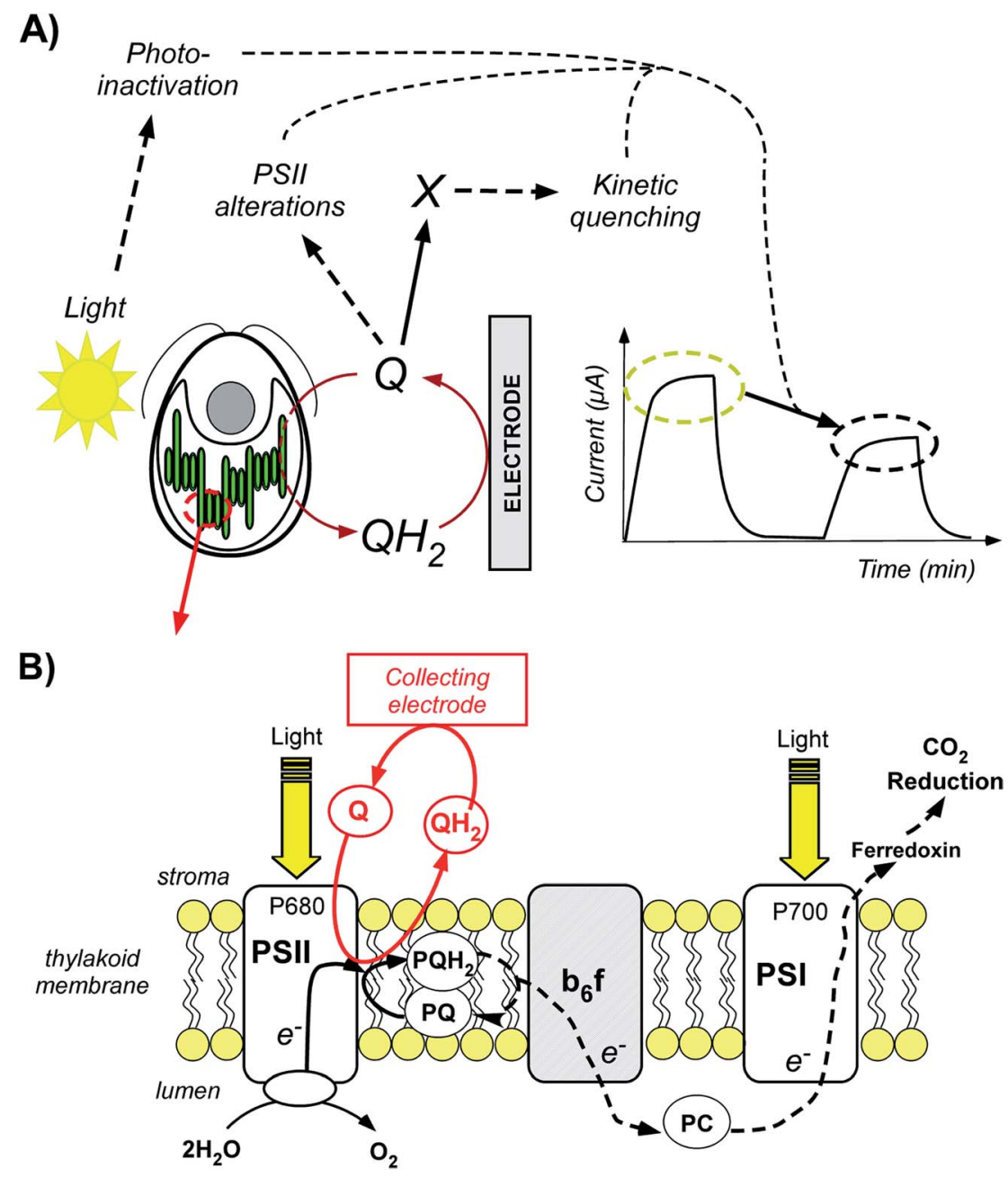

Fig. 1 (A) Scheme of the work reported in this article. Quinones are used to harvest photosynthetic electrons from a green unicellular alga Chlamydomonas reinhardtii and to produce a steady-state photocurrent. Such a current decreased after a second light pulse. This decrease may be related to three phenomena (photoinactivation, production of a new quencher assisted by the quinones, and a direct quinone effect). (B) Overview of photosynthetic electron harvesting at the thylakoid membrane level. Exogenous quinones are used as redox mediators. The oxidized form $\left(Q\right.$, quinone) is reduced by the alga under illumination. The reduced form $\left(\mathrm{QH}_{2}\right.$, hydroquinone) is subsequently oxidized at the collecting electrode surface. The photosynthetic chain contains numerous complexes that transfer electrons from electron donors to acceptors. First, light absorption induces Photosystem II (PSII) excitation. Briefly, light is captured by antenna (LHCII: Light Harvesting Complex II; not shown for more clarity) containing chlorophyll (Chl) and the captured light energy is transferred to the PSIl primary donor, P680 (Chl* + P680 $\rightarrow$ Chl + $\left.\mathrm{P} 680^{*}\right)$. It formally leads to a charge separation which results in water oxidation. Furthermore, in the general case, electron transfer steps occur through the plastoquinone (PQ)/plastoquinol $\left(P \mathrm{PH}_{2}\right)$ pool, the cytochrome $b_{6} f$ complex $\left(b_{6} f\right)$, plastocyanin (PC) and Photosystem I (PSI), eventually leading to $\mathrm{CO}_{2}$ reduction. Solid and dashed lines display the electron flow from PSII (water oxidation) to the final $\mathrm{CO}_{2}$ reduction. In the present work, $\Delta$ petA mutants lacking the $b_{6} f$ complex (displayed in gray) were considered. Therefore, the real electron flow in this case is displayed as a solid line. The red line displays the electron extraction by exogenous quinones as PSII acceptors. 
In this work, we thus wish to investigate these competitive and deleterious phenomena for minimizing them in future applications. By means of electrochemical and fluorescence measurements, we will show that the degradation in terms of performance over time may depend on the experimental conditions and can be attributed to light illumination (photoinactivation) and/or poisoning effects induced by the quinones (Fig. 1A). In the context of photosynthetic microbial fuel cells, this work raises scarcely addressed questions on the long-term effects of quinones and sheds light on this kind of system.

\section{Results and discussion}

\subsection{Time evolution of photocurrent with 2,6-DCBQ}

As previously described, bioelectricity can be produced from a suspension of Chlamydomonas reinhardtii algae in the presence of exogenous quinones (Fig. 1A).$^{35}$ From a mechanistic point of view (Fig. 1B), the quinones (Q) will be prone to access the photosynthetic chain located in the thylakoid membrane inside the chloroplast as they are PSII acceptors. ${ }^{40}$ Under light illumination, Q will undergo reduction by extracting electrons from the level of PSII in the photosynthetic electron transfer chain. The resulting hydroquinone $\left(\mathrm{QH}_{2}\right)$ is then oxidized at the collecting electrode surface $\left(\mathrm{QH}_{2}=\mathrm{Q}+2 \mathrm{e}^{-}+2 \mathrm{H}^{+}\right)$, thus leading to a photocurrent that slowly reaches a quasi-steady state. Fig. 2 displays a representative chronoamperogram recorded during red light illumination (30 $\mathrm{min}$ ) of a suspension of Chlamydomonas reinhardtii $\Delta$ petA algae with 2,6-DCBQ $\left(100 \mu \mathrm{mol} \mathrm{L}^{-1}\right)$. This strain is considered to focus on the electron harvesting at the level of the PSII site (see the Experimental section). One way to estimate the stability of the system is to apply a second light pulse. After the application of a second light pulse, a lower steady-state photocurrent value was measured (Fig. 2). ${ }^{41}$ This is an unexpected result that was not predicted by our previous models that only took into account the redox recycling of quinones by illuminated algae on a preparative scale. ${ }^{35,39}$

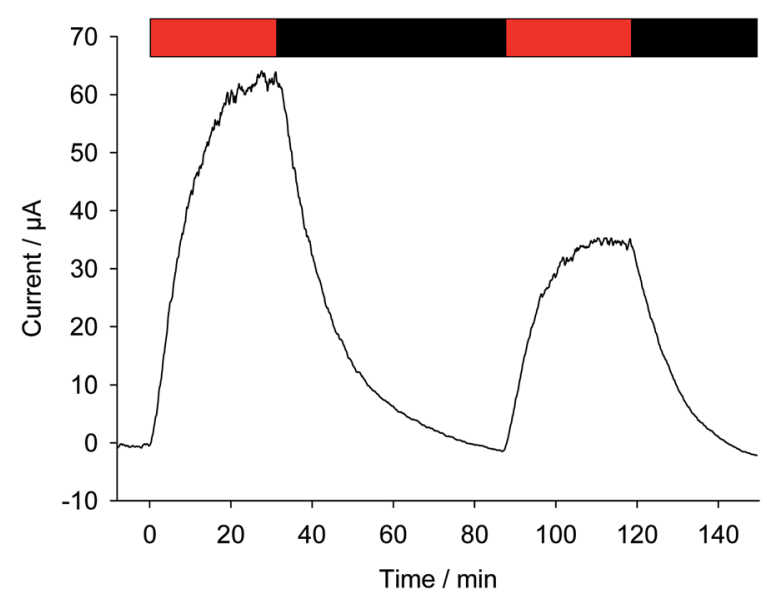

Fig. 2 Current as a function of time (after subtraction of the baseline). Two successive red light pulses have been applied ( $\lambda=640 \mathrm{~nm}$; $340 \mu \mathrm{E} \mathrm{m} \mathrm{m}^{-2} \mathrm{~s}^{-1} ; t=30 \mathrm{~min}$ : red rectangle) to a suspension of Chlamydomonas reinhardtii ( $\Delta$ petA mutants; $10^{7}$ cells per $\mathrm{mL}$ ) in the presence of 2,6 DCBQ $\left(100 \mu \mathrm{mol} \mathrm{L}^{-1}\right)$.
Therefore, this decrease should result from a lower photochemical rate in the biological system (i.e. the rate of quinone reduction by PSII) during the second pulse. This lower photochemical rate should be related to alterations of the biological system during the experiment. On the one hand, algae are under quite strong light conditions during which photoinactivation can occur. For instance, under high-light conditions, Photosystem II cannot fully release its charge due to rate limiting steps in the electron transfer chain that are located downstream of Photosystem II. As a consequence PSII does not reach its maximal turnover rate and photoinhibition (i.e. the formation of reactive species which can damage the photosynthetic chain) can result from the reduced species accumulation. ${ }^{42}$ On the other hand, the algae are continuously incubated with exogenous quinones. Quinones act as redox mediators but could produce deleterious effects as well. Indeed, quinones are often good Michael acceptors that may be able to react (directly or indirectly) with essential macromolecules (e.g. proteins, lipids, and DNA). ${ }^{43,44}$ Thus, further investigation of the effect of quinone incubation on algae in suspension in the dark will be required to fully understand their effects.

\subsection{Effects of quinone incubation}

2.2.1. Principles of fluorescence measurements. More insights into the effects induced by quinones on the algae suspension can be gained by using fluorescence measurements, which are a convenient tool because they provide access to information on the redox state of the PSII site. ${ }^{45-47}$ Indeed, light absorption by PSII leads to excitation of the primary donor P680 (Fig. 3A). Therefore, relaxation by fluorescence competes with electron transfers (involving the primary acceptor $\mathrm{Q}_{\mathrm{A}}$ ) along the photosynthetic chain as well as other relaxation processes such as thermal emission (which includes all quenching phenomena). Fluorescence is thus dependent of the redox state of PSII, i.e. the proportion of open (with oxidised $\mathrm{Q}_{\mathrm{A}}$ ) and closed (with reduced $\mathrm{Q}_{\mathrm{A}}{ }^{-}$) centers. A typical fluorescence analysis is performed according to the following steps (Fig. 3B): ${ }^{37}$ (1) a first continuous illumination increases the initial fluorescence level $F_{0}$ to a fluorescence value $F_{\text {stat }} . F_{0}$ is the minimum fluorescence level when all PSII centers are in an open state (as defined in Fig. 3A). $F_{\text {stat }}$ corresponds to a steady-state fluorescence value that assesses the photosynthetic activity occurring with a certain PSII photochemical conversion capacity, which is controlled by the proportion of open and closed centers; (2) a second supersaturating pulse is then applied and produces the maximum fluorescence level $F_{\max }$. This light pulse of $250 \mathrm{~ms}$ is long enough to fully reduce all electron acceptors downstream of PSII, thus leading to the full reduction of $\mathrm{Q}_{\mathrm{A}}$ to $\mathrm{Q}_{\mathrm{A}}{ }^{-}$. $F_{\text {max }}$ thus corresponds to all PSII centers being in a closed state (as defined in Fig. 3A) where the PSII photochemical conversion capacity is zero. The proportion of photons that are converted during photosynthetic activity is thus proportional to $F_{\max }-$ $F_{\text {stat }}$.

The ratio of open centers $\Phi$ is defined as the proportion of oxidized PSII sites. It can be calculated with the $F_{0}, F_{\text {stat }}$ and $F_{\text {max }}$ values $\left(\Phi=\left(F_{\max }-F_{\text {stat }}\right) /\left(F_{\max }-F_{0}\right)\right)$ and yields the redox state of 
A)

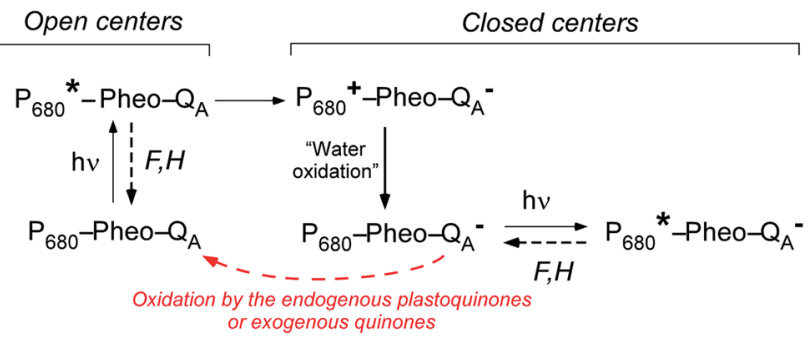

B)

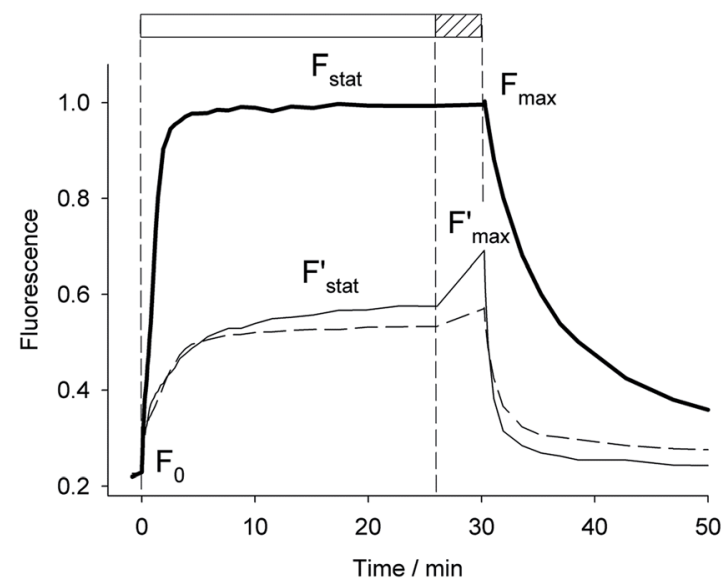

Fig. 3 (A) Overview of the different pathways within Photosystem II after light excitation. Relaxation can occur by fluorescence (F) or heat $(\mathrm{H})$ emission (such pathways mainly include relaxations of excited chlorophyll antenna; see the Fig. 1 legend. For more clarity, only the decays from the excited state of P680 are displayed). Additionally, a charge separation occurs from the reduction of the primary acceptor $\mathrm{Q}_{\mathrm{A}}$ by $\mathrm{P} 680$ through pheophytin (Pheo). The oxidized $\mathrm{P} 60^{+}$then induces the oxidation of water. The electron transfer along the chain will then proceed by means of the plastoquinone pool (see Fig. 1) Exogenous quinones are PSII acceptors and can extract photosynthetic electrons by oxidizing $\mathrm{Q}_{\mathrm{A}}{ }^{-}$or the plastoquinone pool. (B) Fluorescence induction curves (see the text) achieved with a suspension of Chlamydomonas reinhardtii ( $\Delta$ petA mutants; $10^{7}$ cells per $\mathrm{mL}$ ). The solid lines correspond to typical curves without any addition of exogenous quinones (solid thick line) or in the presence of 2,6 DCBQ (solid thin and dashed lines; $25 \mu \mathrm{mol} \mathrm{L}{ }^{-1}$ ). The thin solid line corresponds to a curve recorded just after the addition of quinones. The dashed line displays the induction curve after $3 \mathrm{~h}$ of pre-incubation with the quinone. The fluorescence values are normalized with respect to the maximum value $F_{\max }$. The shape of the induction curve is related to the illumination sequence. Firstly, a continuous illumination is applied (top rectangle in white). The initial fluorescence level, $F^{\circ}$, is related to a situation in which all the $Q_{A}$ centers are in an oxidized state ((A) left). Then, the photosynthetic activity begins. Some open $Q_{A}$ centers are reduced to closed $Q_{A}{ }^{-}$centers which may be oxidized by endogenous plastoquinones or exogenous quinones ((A) right). This leads to a higher steady-state fluorescence level $\left(F_{\text {stat }}\right)$ that results from the relaxation of open $\left(Q_{A}\right)$ and closed $\left(Q_{A}{ }^{-}\right)$centers $((A)$ left and right). Secondly, the supersaturating pulse (top hatched rectangle) prevents the oxidation of $\mathrm{Q}_{\mathrm{A}}{ }^{-}$, thus leading to a maximum fluorescence level $\left(F_{\text {max }}\right)$.

the PSII acceptors. ${ }^{37,38}$ As reported in previous studies, this is a good estimate of an exogenous PSII acceptor efficiency. ${ }^{47}$ As an example, adding exogenous quinones to the algae suspension leads to an increase in $\Phi$, due to electron transfer between the reduced PSII sites and the added quinones (Fig. $3 \mathrm{~A}) \cdot{ }^{37,38}$ In this work, we focus on the PSII state by means of the $\Delta$ petA mutant which is devoid of a major electron carrier, the cytochrome $b_{6} f$ complex, downstream of the plastoquinone pool which is the reservoir of electron acceptors for PSII. In the absence of exogenous quinones, the electron transfer chain is thus interrupted downstream of PSII, so $F_{\max }$ and $F_{\text {stat }}$ values are very similar, thus leading to $\Phi \sim 0$. Additionally, quinones are also able to perform fluorescence quenching by interacting directly with excited chlorophyll (the chlorophyll excitation $(\mathrm{Chl}+h \nu \rightarrow$ $\left.\mathrm{Chl}^{*}\right)$ is followed by an electron transfer from the excited state of chlorophyll to quinone and the formation of a charge transfer complex $\left(\mathrm{Chl}^{*}+\mathrm{Q} \rightarrow\left[\mathrm{Chl}^{*} \ldots \mathrm{Q}\right] \rightarrow\left[\mathrm{Chl}^{+}, \mathrm{Q}^{-}\right]\right)$. In apolar media like thylakoid membranes, the separation of the ion pair is not favored and the charge transfer complex is rather assumed to decay to the ground state: $\left.\left[\mathrm{Chl}^{+}, \mathrm{Q}^{-}\right] \rightarrow \mathrm{Chl}+\mathrm{Q}\right) \cdot{ }^{\mathbf{4 8 , 4 9}}$ Following the Stern-Volmer law, this quenching can be estimated by using maximum fluorescence levels before and after quinone addition $\left(F_{\max }\right.$ and $F_{\max }^{\prime}$ respectively) according to $\left(F_{\max }-F_{\max }^{\prime}\right) / F_{\max }^{\prime}$.

2.2.2. Quenching studies. Sequential fluorescence measurements were thus performed to assess the long-term effect of quinone incubation. As displayed in Fig. 3B, fluorescence curves are modified after adding 2,6-DCBQ as an exogenous quinone. At very short times, $F_{\text {stat }}$ and $F_{\max }$ values (thick and thin solid lines) decreased. These decreases are consistent with the ability of 2,6-DCBQ to harvest electrons from PSII and to perform fluorescence quenching, respectively. ${ }^{37,38}$ However, even after $3 \mathrm{~h}$ under dark conditions, $F_{\text {stat }}$ and $F_{\max }$ values continue to decrease. Fig. 4 displays the quenching parameter $\left(F_{\max }-F_{\max }^{\prime}\right) / F_{\max }^{\prime}$ as a function of time. It confirms that the fluorescence quenching endured by the algae progressively increases during quinone incubation (Fig. 4). These experimental results suggest that, assuming that chlorophylls are not altered, an additional (and not light dependent) quenching is induced by the exogenous quinones during long-term incubation. ${ }^{50}$ This led us to consider the presence of a second fluorescence quencher (named $\mathrm{X}$, concentration $C_{\mathrm{X}}$ ) that could be slowly produced from a process initiated by the quinone (named $\mathrm{Q}$, concentration $C_{\mathrm{Q}}$ ) during the incubation (like the denaturation of a biological entity). From the excitation/ relaxation pathways summarized in Fig. 5A, a corresponding mathematical model can be established, one that relies on the simplified scheme displayed in Fig. 5B. It takes into account how the fluorescence quencher could modify the fluorescence levels related to the intact photosynthetic mechanism. Globally, the usual pathways following the excited P680 (fluorescence $k_{\mathrm{F}}$, heat $k_{\mathrm{T}}$, quenching by quinones $k_{\mathrm{Q}}$ ) are complemented with a new quenching induced by species $\mathrm{X}$. Using the quasi-steady state approximation applied for the excited state $\mathrm{P}^{*} 680$, the fluorescence $F_{\text {max }}^{\prime}$ (in the presence of quinones) is expressed by eqn (1):

$$
F_{\text {max }}^{\prime}=\frac{k_{\mathrm{F}} f(I)}{f(I)+k_{\mathrm{F}}+k_{\mathrm{T}}+k_{\mathrm{Q}} C_{\mathrm{Q}}+k_{\mathrm{X}} C_{\mathrm{X}}}
$$

where $f(I)$ is the rate constant of $\mathrm{P} 680$ excitation (resulting from an incident light flux $I$ ), $k_{\mathrm{T}}$ and $k_{\mathrm{F}}$ are the rate constants corresponding to the relaxation by heat and fluorescence production, 


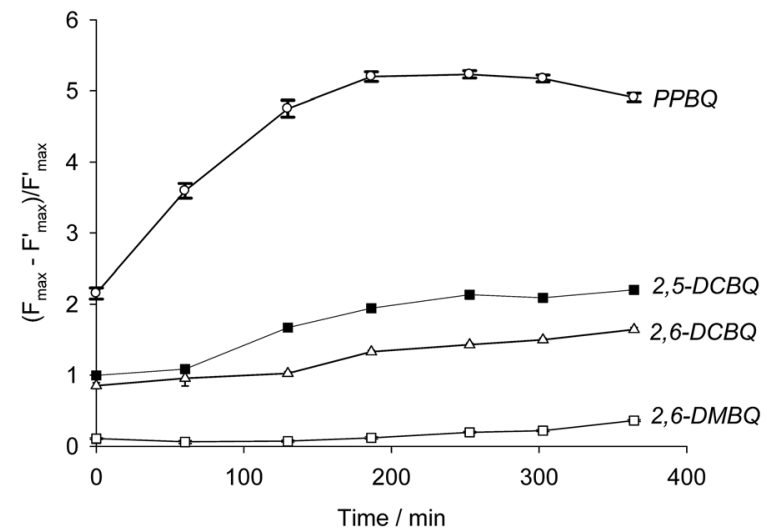

Fig. 4 Quenching parameter as a function of incubation time for four exogenous quinones $\left(C_{Q}=25 \mu \mathrm{mol} \mathrm{L}^{-1}\right)$ : PPBQ (white circles), 2,5-DCBQ (black squares), 2,6-DCBQ (white triangles) and 2,6-DMBQ (white squares).

respectively, and $k_{\mathrm{Q}}$ and $k_{\mathrm{x}}$ are the rate constants for the quenching by the exogenous quinone, $\mathrm{Q}$, and $\mathrm{X}$, respectively. When no exogenous quinones are added, $C_{\mathrm{Q}}=C_{\mathrm{X}}=0$. Thus eqn (1) becomes eqn (2) and defines $F_{\max }$, i.e. the maximum fluorescence level after the supersaturating pulse without any quinone effect:

$$
F_{\max }=\frac{k_{\mathrm{F}} f(I)}{f(I)+k_{\mathrm{F}}+k_{\mathrm{T}}}
$$

Considering both eqn (1) and (2) leads to eqn (3):

$$
\frac{F_{\max }-F_{\max }^{\prime}}{F_{\max }^{\prime}}=K_{\mathrm{Q}} C_{\mathrm{Q}}+K_{\mathrm{X}} C_{\mathrm{X}}
$$

Two constants, $K_{\mathrm{Q}}$ and $K_{\mathrm{X}}$, are defined below and correspond to quenching constants for $\mathrm{Q}$ and $\mathrm{X}$ respectively:

$$
\begin{aligned}
& K_{\mathrm{Q}}=\frac{k_{\mathrm{Q}}}{f(I)+k_{\mathrm{T}}+k_{\mathrm{F}}} \\
& K_{\mathrm{X}}=\frac{k_{\mathrm{X}}}{f(I)+k_{\mathrm{T}}+k_{\mathrm{F}}}
\end{aligned}
$$

The $\left(F_{\max }-F_{\max }^{\prime}\right) / F_{\max }^{\prime}$ parameter is expressed as a global quenching parameter that can be calculated from the fluorescence measurements introduced above. In the present case, this parameter depends on both quinone and quencher concentrations, $C_{\mathrm{Q}}$ and $C_{\mathrm{X}}$. It thus takes into account the two kinds of quenching. The first constant, already published elsewhere, is the instantaneous quenching due to quinones under light experiments. ${ }^{37}$ The second constant, which depends on the concentration of $\mathrm{X}$, is related to a kinetic quenching due to the quinone incubation (and the subsequent formation of $\mathrm{X}$ ) during a given dark period. As a consequence, a new quenching term, named $Q_{\mathrm{x}}$, can be defined and viewed as a kinetic quenching that can be deduced from the time-dependent global quenching $\left(\left(F_{\max }-F_{\max }^{\prime}\right) / F_{\max }^{\prime}\right)$ by subtracting the initial quenching due to the quinone.
A)

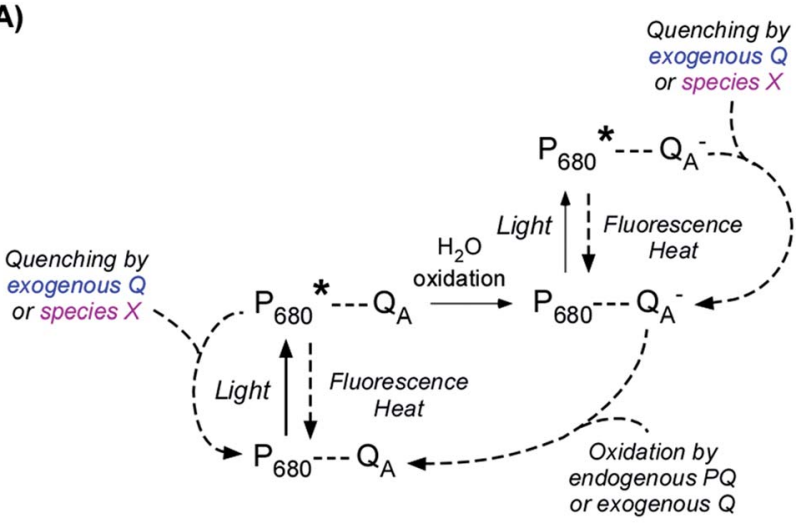

Denaturation of a biological entity $P$ induced by exogenous quinones $Q$

$$
\mathrm{P} \stackrel{\mathrm{Q}}{\longrightarrow} \mathrm{X}
$$

B)

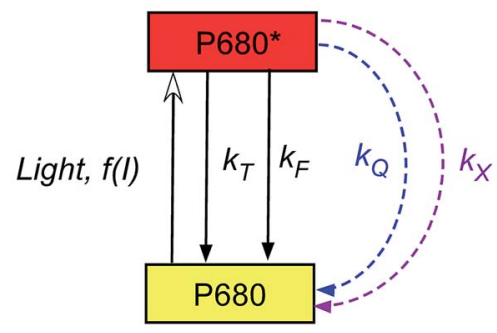

Fig. 5 (A) Overview of the main relaxation pathways occurring from an excited PSII and related to the $F_{\max }$ level changes when exogenous quinones $Q$ are added. Please note that exogenous quinones are efficient quenchers through their interaction with excited chlorophyll (Chl*) from the PSII antennae upstream of the excited P680 site. For simplification, the quenching from exogenous $Q$ and species $X$ is shown at the $\mathrm{P} 680^{*}$ site. (B) Simplified scheme summarizing the above overview and used for modeling the kinetic quenching. $f(I)$ is the rate constant of $\mathrm{P} 680 \rightarrow \mathrm{P} 680 *$ excitation (resulting from an incident light flux I). $k_{\mathrm{T}}$ and $k_{\mathrm{F}}$ are the rate constants corresponding to relaxations by heat and fluorescence production, respectively. Dashed arrows are related to the quenching induced by exogenous quinones. $k_{\mathrm{Q}}$ is the rate constant due to the direct and usual quenching by the exogenous quinone (blue). The rate constant $k_{\mathrm{X}}$ is related to the dynamic quenching (purple) induced by the new quenching species $X$ (see the text).

$$
\begin{aligned}
Q_{\mathrm{X}} & =\frac{F_{\max }-F_{\max }^{\prime}}{F_{\max }^{\prime}}-K_{\mathrm{Q}} C_{\mathrm{Q}} \\
& =\left(\frac{F_{\max }-F_{\max }^{\prime}}{F_{\max }^{\prime}}\right)-\left(\frac{F_{\max }-F_{\text {max }, t=0}^{\prime}}{F_{\text {max }, t=0}^{\prime}}\right)=K_{\mathrm{X}} C_{\mathrm{X}}
\end{aligned}
$$

By assuming that quencher $\mathrm{X}$ results from the denaturation of a biological entity through a first order reaction (involving a rate constant $k$ ), the concentrations of this entity and $\mathrm{X}$ can be expressed as $\mathrm{e}^{-k t}$ and $1-\mathrm{e}^{-k t}$, respectively. Eqn (7) can be eventually written as

$$
Q_{\mathrm{X}}=K_{\mathrm{X}} C_{\mathrm{X}}=K_{\mathrm{X}}\left(1-e^{-k t}\right)
$$


Modeling using eqn (7) was thus attempted from the experimental data. The results are summarized in Table 1 and Fig. 6. In the case of PPBQ, a quite good agreement is observed between the theoretical curve and the experimental data. The kinetic quenching $Q_{\mathrm{X}}$ appears relatively fast and saturates at a value of $K_{\mathrm{X}}=3.0 \pm 0.1$. In other cases, the kinetic quenching is unfortunately too slow to perform reliable calculations of the quenching constant $K_{\mathrm{X}}$ from the fluorescence measurements (Fig. 6). Nevertheless, according to the modeling reported here, the kinetic quenching does not result from the exogenous quinones themselves but from a biological degradation product. In other words, the quenching constant $K_{\mathrm{X}}$ should be quite similar for all the investigated quinones. This assumption can be used to fit the data for 2,5-DCBQ or 2,6-DCBQ using this value of $K_{\mathrm{X}}$ and eqn (7), and thus to deduce the rate constant $k$ for both cases (Table 1). Interestingly, in the 2,6-DMBQ case (i.e. a redox mediator with low efficiency to harvest electrons from $\mathrm{PSII}^{37}$ ), the kinetic quenching is particularly slow, thus leading to a peculiar data treatment to calculate the rate constant $k$ (see ESI Fig. S1†).

2.2.3. Analysis of the ratio of open centers. The analysis of the quenching parameter can be enriched by an analysis of the evolution of the fraction of open centers over time (Fig. 7). In order to compare all the quinones, a semi-logarithmic scale is used to analyze the variations of $\Phi$ as a function of time.

Table 1 Rate constants extracted from the fitting of the kinetic quenching $Q_{x}$ using eqn (7)

\begin{tabular}{lll}
\hline Added quinones & $k\left(\mathrm{~s}^{-1}\right)$ & $R^{2}$ \\
\hline PPBQ & $(2.4 \pm 0.6) \times 10^{-4}$ & 0.97 \\
$2,5-\mathrm{DCBQ}$ & $(2.5 \pm 0.2) \times 10^{-5}$ & 0.95 \\
$2,6-\mathrm{DCBQ}$ & $(1.4 \pm 0.1) \times 10^{-5}$ & 0.97 \\
$2,6-\mathrm{DMBQ}$ & $(6.0 \pm 1.0) \times 10^{-6}$ & 0.96
\end{tabular}

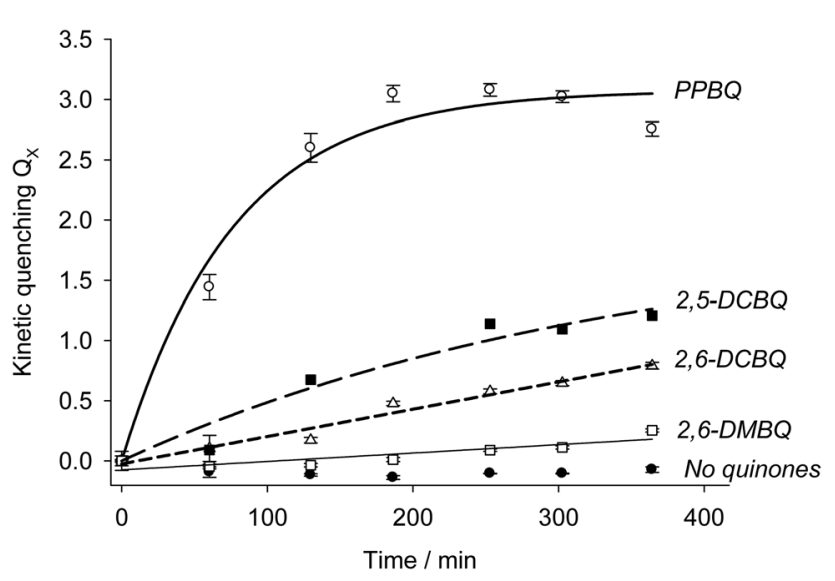

Fig. 6 Kinetic quenching as a function of incubation time in the presence of PPBQ (white circles), which leads to a final constant $K_{\mathrm{X}}$ value of $(3.0 \pm 0.1)$. The corresponding $k$ rate constant is $(2.4 \pm 0.6) \times$ $10^{-4} \mathrm{~s}^{-1}$ (see the text). Other exogenous quinones were investigated: 2,5-DCBQ (black squares), 2,6-DCBQ (white triangles) and 2,6-DMBQ (white squares). Control experiments in the absence of quinones are displayed with black circles. In this case, the corresponding rate constant $(k)$ is calculated by using the same $K_{\mathrm{x}}$ value for each quinone.

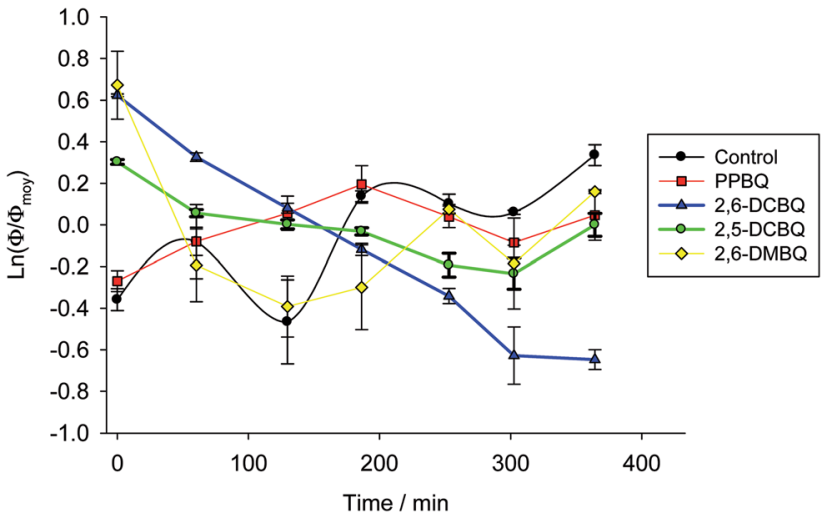

Fig. 7 Fraction of open centers $(\Phi)$ as a function of incubation time with several quinones: PPBQ (red squares), 2,5-DCBQ (green circles), 2,6-DCBQ (blue triangles), 2,6-DMBQ (yellow diamonds), and the control without any quinone (black circles). For ease of comparison, $\Phi$ is normalized by its average value over time ( $\Phi_{\text {moy; }}$ see the text) and is displayed by using a semi-logarithmic scale.

Additionally, the proportion of open centers for a given quinone is also normalized with respect to its average value $\Phi_{\text {moy }}$ throughout the corresponding experiment. Of note, the data randomly fluctuate in the case of control and DMBQ experiments. This is due to the intrinsically low values of the ratio of open centers in both cases which results in higher uncertainties. Globally, for 2,6-DMBQ and PPBQ, the fraction of open centers does not strongly depend on the time of incubation under dark conditions. Conversely, the chloroquinones (2,5-DCBQ and especially 2,6-DCBQ) led to a decrease of the open center ratio under the same conditions. Therefore, a more detailed analysis with different 2,6-DCBQ concentrations (from 25 to $100 \mu \mathrm{mol} \mathrm{L} \mathrm{L}^{-1}$ ) was performed. First of all, the kinetic quenching for 2,6-DCBQ was investigated through a semilogarithmic analysis (Fig. S2 $\dagger$ ) in agreement with eqn (6). Apparent rate constants can thus be extracted (Table S1 $\dagger$ ) and show that quencher $\mathrm{X}$ is produced faster for high 2,6-DCBQ concentrations. Furthermore, another semi-logarithmic analysis also ascertains that 2,6-DCBQ alters the open center ratio over time and allows one to extract apparent rate constants related to the decrease of the fraction of open centers (Fig. S3 and Table S2 $\dagger$ ).

2.2.4. Discussion. A new kinetic quenching is revealed for all the investigated quinones. 2,6-DCBQ and PPBQ, the two best PSII acceptors under these conditions,${ }^{37}$ did not have the same behavior, suggesting that there are important differences between the two quinones. 2,6-DCBQ leads to moderate kinetic quenching and the decrease of the proportion of open centers without any observed saturation at this timescale. In the case of PPBQ, the kinetic quenching appears faster and leads to a saturation in 2 hours without any clear effect on $\Phi$. In the case of 2,6-DCBQ at least, this kinetic quenching $Q_{\mathrm{x}}$ is combined with an open site fraction decrease. The open site fraction $\Phi$ is usually an increasing function of the quinone concentration $C_{\mathrm{Q}}$ if only considering the harvesting behavior. ${ }^{37,38}$ The decrease in open center ratio is thus a supplementary effect that reveals a second pathway of alteration possibly induced by the quinone 
itself (maybe the usual cytotoxicity of quinones by reaction with essential macromolecules ${ }^{\mathbf{4}}$ ) in addition to the kinetic quenching.

The differences in quinones can largely be summarized by electrophilicity. Taking PPBQ, DCBQs and 2,6-DMBQ as examples and the midpoint potentials $\left(\mathrm{Q} / \mathrm{QH}_{2}\right)$ at $\mathrm{pH} 7$ that have been reported in the literature (Scheme 1), ${ }^{37,51,52}$ we can find a general trend in electrophilicity according to a higher $E^{\circ \prime}$ value: $^{53}$ DCBQs, PPBQ, and DMBQ (from highest to lowest). Thus, DCBQs are more able to react with nucleophiles than PPBQ, which exhibits better partitioning into membranes and may quench chlorophylls directly (because this phenomenon occurs within membranes, i.e. in a hydrophobic medium). On the other hand, 2,6-DMBQ is less electrophilic and less lipophilic, ${ }^{37}$ and is thus less likely to lead to kinetic quenching or an alteration of PSII. Despite its relatively poor ability to accept electrons from PSII, ${ }^{37}$ this suggests a further investigation of its use in photoelectrochemical fuel cells in which the electron harvesting will be moderate when remaining stable over time.

It is of note that kinetic quenching seems to occur faster for PPBQ when compared to the other quinones. Because PPBQ is the highest chlorophyll fluorescence quencher and the most expected lipophilic molecule among all the investigated quinones, ${ }^{37}$ it may suggest that the kinetic quenching is also related to partitioning phenomena, in contrast to the electron harvesting that may be mainly controlled in this range of concentration by electron transfer rather than mass transport. ${ }^{37,38}$ It thus raises the question if the kinetic quenching is also related to the progressive diffusion of exogenous quinones over time. This cannot be fully excluded but our data about the chlorophyll quenching by quinones (this work and ref. 37 and 38) suggest that the timescale of quinone diffusion is shorter than that of our experiments. This thus supports the assumption of a new quencher formation rather than that of the slow diffusion of quinones within the chloroplast.<smiles>O=C1C=C(Cl)C(=O)C(Cl)=C1</smiles>
(2,6-DCBQ)

$E^{\circ \prime}(p H 7)=315 m V$ vs SHE<smiles>O=C1C=C(Cl)C(=O)C=C1Cl</smiles>

2,5-Dichlorobenzoquinone (2,5-DCBQ)

$E^{\circ 1}(\mathrm{pH} 7)=309 \mathrm{mV}$ vs SHE
2,6-Dichlorobenzoquinone<smiles>O=C1C=CC(=O)C(c2ccccc2)=C1</smiles>

p-phenylbenzoquinone (PPBQ)

$E^{\circ}(\mathrm{pH} 7)=277 \mathrm{mV}$ vs SHE<smiles>CC1=CC(=O)C=C(C)C1=O</smiles>

2,6-Dimethylbenzoquinone (2,6-DMBQ)

$E^{\circ \prime}(p H 7)=174 m V$ vs SHE
Scheme 1
Additionally, it is worth mentioning that the reported effects probably resulted from the oxidized form $\mathrm{Q}$ rather than from oxidative stress induced by reduced forms like hydroquinone $\mathrm{QH}_{2}$ or semiquinone $\mathrm{Q}^{-}$. If considering the redox potential of the $\mathrm{O}_{2} / \mathrm{O}_{2}{ }^{-}$couple $(-155 \mathrm{mV}$ vs. SHE), redox potentials of the $\mathrm{Q} / \mathrm{QH}_{2}$ and $\mathrm{Q} / \mathrm{Q}^{-}$couples are too positive to lead to the formation of the superoxide anion through the reaction between reduced forms of quinones and dioxygen. ${ }^{54}$ Furthermore, similar experiments were performed with the hydroquinone form. No large effect was observed under these conditions (see Fig. S4 in the ESI $\dagger$ ).

To summarize, we figured out two possible side effects of quinones (production of a second quencher $\mathrm{X}$ and alteration of the fraction of PSII open centers). Additionally, the expected PSII accepting behavior may be related to the decrease of the steady-state photocurrent after the second light pulse for 2,6DCBQ. Because the kinetic quenching rapidly saturates without any changes of the ratio of open centers, PPBQ is a suitable quinone for investigating the effects of successive light illuminations on photocurrents under conditions where the system no longer evolves by side-effects of quinones.

\subsection{Photocurrents as a function of time with PPBQ}

2.3.1. Comparison between PPBQ and 2,6-DCBQ. Fig. 8 displays four successive photocurrents recorded under quite high light conditions from a suspension of Chlamydomonas reinhardtii with a long $\mathrm{PPBQ}$ pre-incubation time (8 hours) at high concentration $\left(100 \mu \mathrm{mol} \mathrm{L}^{-1}\right)$. Under these conditions, as shown above, the kinetic quenching reached a saturation level with PPBQ. As a consequence, a photocurrent decrease between two pulses can only result from photoinactivation. Thus, in contrast to 2,6-DCBQ (same quinone concentration and incident light values, see Fig. 2), each photocurrent magnitude slowly decreased..$^{55}$ More precisely, comparisons between PPBQ and 2,6-DCBQ (current magnitude and area, see Table 2)

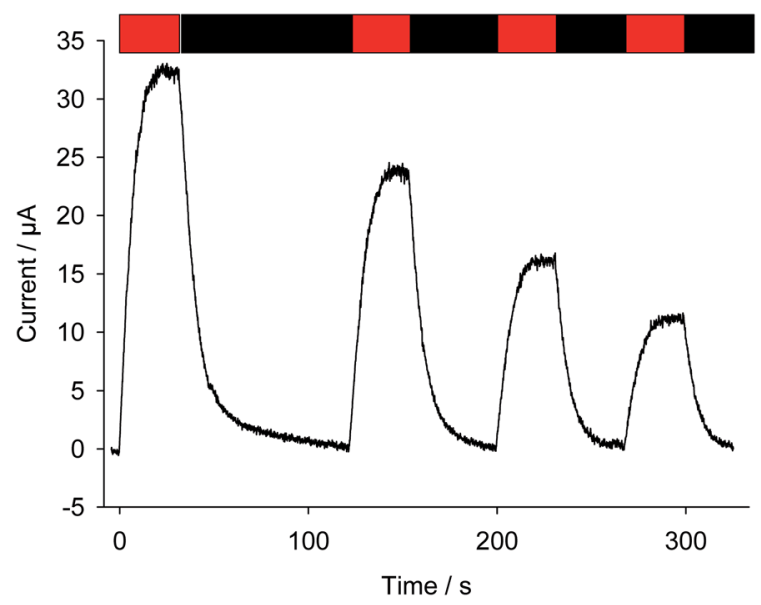

Fig. 8 Photocurrents as a function of time (after subtraction of the baseline). Four successive light pulses have been applied ( $\lambda=640 \mathrm{~nm}$; $340 \mu \mathrm{E} \mathrm{m}^{-2} \mathrm{~s}^{-1} ; 30$ min duration: red rectangle) to a suspension of Chlamydomonas reinhardtii $\Delta$ petA mutants $\left(10^{7}\right.$ cells $\left.\mathrm{mL}^{-1}\right)$ in the presence of PPBQ $\left(100 \mu \mathrm{mol} \mathrm{L}{ }^{-1}\right)$ after 8 hours of pre-incubation. 
Table 2 Peak currents and area resulting from photocurrents recorded $(n=3)$ with 2,6-DCBQ and PPBQ under red light conditions (see the text). Data extracted from Fig. 2 (2,6-DCBQ) and Fig. 8 (PPBQ)

\begin{tabular}{|c|c|c|c|c|c|c|c|c|}
\hline & Current $(\mu \mathrm{A})$ & Decrease (\%) & Area $(\mu \mathrm{C})$ & Decrease (\%) & Current $(\mu \mathrm{A})$ & Decrease (\%) & Area $(\mu \mathrm{C})$ & Decrease (\%) \\
\hline Pulse 2 & $35.3 \pm 3.4$ & 45 & $(6.77 \pm 0.70) \times 10^{4}$ & 49 & $24.6 \pm 2.5$ & 26 & $(4.73 \pm 0.40) \times 10^{4}$ & 34 \\
\hline Pulse 3 & - & - & - & - & $16.8 \pm 2.0$ & 32 & $(3.14 \pm 0.30) \times 10^{4}$ & 34 \\
\hline Pulse 4 & - & - & - & - & $11.7 \pm 1.0$ & 31 & $(2.14 \pm 0.20) \times 10^{4}$ & 32 \\
\hline
\end{tabular}

provide evidence to show that the current after several pulses decreases more for 2,6-DCBQ ( $\sim 50 \%)$ than for PPBQ ( 30\%). This is globally consistent with the fact that only photoinactivation occurs under these conditions when PPBQ is used because kinetic quenching saturates after $4 \mathrm{~h}$ (see Fig. 6). Conversely, the photocurrent decrease with 2,6-DCBQ was more significant and was affected by both photoinactivation and sideeffects of quinones (kinetic quenching + PSII alterations). While the photocurrent decrease is comparable in terms of magnitude to the evolution of kinetic quenching as assessed by fluorescence measurements (10 000-15 $000 \mathrm{~s}$ ), a more accurate analysis remains difficult to achieve since making comparisons for both quinones under the same conditions is not strictly feasible.

2.3.2. PPBQ under moderate illumination conditions. In order to further discriminate effects resulting from quinones (PSII alterations or kinetic quenching) and photoinactivation, experiments were carried out under moderate illumination conditions (green light; see Fig. 9) with PPBQ at a low preincubation time $(1 \mathrm{~h})$ and with a high concentration $\left(100 \mu \mathrm{mol} \mathrm{L}{ }^{-1}\right)$. The corresponding electrochemical measurements show that lower photocurrents were recorded in comparison with the previous experiment (high pre-incubation time - high light illumination). Of note, the photocurrent decrease is only observed between the first two pulses and the

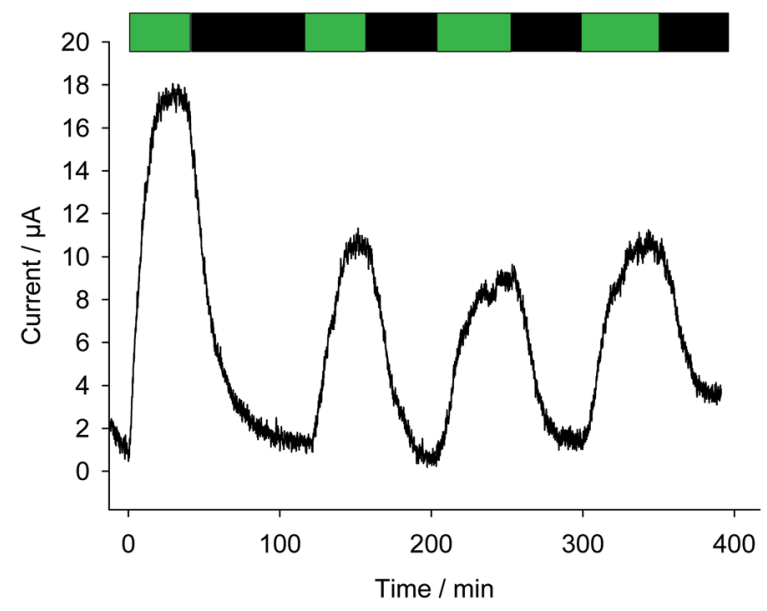

Fig. 9 Four successive photocurrents as a function of time (after subtraction of the baseline) under green light pulses $(\lambda=530 \mathrm{~nm}$; green bands) with a suspension of Chlamydomonas reinhardtii $\Delta$ petA mutants $\left(10^{7}\right.$ cells per $\left.\mathrm{mL}\right)$ in the presence of PPBQ $\left(100 \mu \mathrm{mol} \mathrm{L}^{-1}\right)$ after a pre-incubation time of $1 \mathrm{~h}$. photocurrent magnitude does not exhibit further changes for the following pulses. These results suggest that the kinetic quenching could only be involved in the decrease of photocurrent between the first two pulses. Indeed, under these light conditions, photoinactivation is expected to be less significant since PPBQ did not show any effect towards PSII activity. Therefore, kinetic quenching progressively takes place at the beginning of the experiment. During this period, kinetic quenching reaches a saturation level (as evidenced by the fluorescence measurements) and should no longer occur. This is confirmed by the constant photocurrent intensity for the following pulses. The time period of the first two light pulses corresponds to the photocurrent decrease and is globally comparable to that which is required to reach a constant kinetic quenching through fluorescence measurements. This therefore suggests that for $\mathrm{PPBQ}$, the kinetic quenching is possibly involved in the drop of performance at least at relatively short timescales.

This trend is quantitatively confirmed by complementary fluorescence measurements. As evidenced above, the maximum kinetic quenching value $K_{\mathrm{X}}$ is close to 3 . Furthermore, the maximum fluorescence level following the supersaturating pulse without any kinetic quenching, ${F^{\prime}}_{\max }^{*}$, can be expressed as follows:

$$
F_{\max }^{\prime}{ }^{*}=\frac{k_{\mathrm{F}} f(I)}{f(I)+k_{\mathrm{F}}+k_{\mathrm{T}}+k_{\mathrm{Q}} C_{\mathrm{Q}}}
$$

A new kinetic quenching parameter can thus be written:

$$
\frac{{F^{\prime}}_{\text {max }}^{*}-F_{\text {max }}^{\prime}}{F_{\text {max }}^{\prime}}=K_{\mathrm{X}}^{*} C_{\mathrm{X}}
$$

With a $K_{\mathrm{X}}^{*}$ constant defined as

$$
K_{\mathrm{X}}^{*}=\frac{k_{\mathrm{X}}}{f(I)+k_{\mathrm{F}}+k_{\mathrm{T}}+k_{\mathrm{Q}} C_{\mathrm{Q}}}
$$

Combining eqn (5) and (10) leads to

$$
\frac{K_{\mathrm{X}}}{K_{\mathrm{X}}^{*}}=1+\frac{k_{\mathrm{Q}} C_{\mathrm{Q}}}{f(I)+k_{\mathrm{F}}+k_{\mathrm{T}}}=1+K_{\mathrm{Q}} C_{\mathrm{Q}}
$$

The $K_{\mathrm{Q}}$ value has already been determined $((2.37 \pm 0.19) \times$ $10^{4} \mathrm{~L} \mathrm{~mol}^{-1}$ ) previously. ${ }^{37}$ Under our conditions, if taking into account the lost quinone amount in other compartments 
$\left(14.3 \mu \mathrm{mol} \mathrm{L}^{-1}\right.$; see details in ref. 37$), K_{\mathrm{Q}} C_{\mathrm{Q}}$ is close to 2 and helps calculate the $K_{\mathrm{x}}^{*}$ value which is eventually equal to 1 . Moreover, eqn (9) shows that $F_{\max }^{\prime}$ tends to ${F^{\prime}}^{*}{ }_{\max } /\left(1+K_{\mathrm{X}}^{*}\right) \sim 0.5{F^{\prime}}^{\prime *}$ max when saturation is reached $\left(C_{\mathrm{X}} \rightarrow 1\right)$. Assuming a correlation between the steady-state photocurrent and the kinetic quenching in the case of PPBQ under moderate illumination, it thus means that the initial photocurrent (first pulse) should be two times higher than the photocurrent recorded after saturation of the poisoning (second pulse). Considering a magnitude of $10 \mu \mathrm{A}$ during the second pulse, the first photocurrent intensity would be close to $20 \mu \mathrm{A}$, i.e. close to the experimental value of $17 \mu \mathrm{A}$ (the slight discrepancy may be explained by the fact that the kinetic quenching effect started before the first pulse). However, we cannot exclude that photoinactivation still occurs during the first two pulses. Indeed, photoinactivation is usually related to the photochemical rate (a high photochemical rate should induce more photoinactivation). Thus, during the first pulse and the higher photocurrent, the photoinactivation rate may be quite high, thus preventing the efficacy of reparation pathways, subsequently decreasing the photochemical rate such that it would not be high enough to induce a significant photoinactivation during the next light pulses.

To summarize, these experiments globally confirmed that three phenomena can explain the photocurrent decrease at relatively long timescales. Of note, under moderate illumination conditions with PPBQ over long timescales, the photocurrent remains constant after successive pulses. These peculiar conditions (no photoinactivation, saturated kinetic quenching and no alteration of open centers) allow one to examine photosynthetic electron harvesting only controlled by an electrocatalytic mechanism involving Michaelis-Menten kinetics. ${ }^{35,39}$

2.3.3. The dark current. The above results suggest that working at low quinone incubation time and low excitation light should allow one to control and limit the degradations of the system to produce a more stable current. In this case, it will require recording the photocurrent as fast as possible after quinone introduction, i.e. when the baseline is not yet stable. Indeed under our conditions (i.e. when quinones are in the presence of algae), a dark current appears and this has been attributed to interaction with mitochondria. ${ }^{35,37}$ This current first increases and then diminishes until stabilization. If illumination is quickly triggered, the photocurrent will be recorded at very short quinone incubation time but the dark current will not be stable during the light pulse. Without any stable baseline return, the actual photocurrent will be calculated with difficulty from the whole measured current and could lead to possible misinterpretations. Thus, a compromise has to be found between working on a less impaired system and being able to precisely distinguish the photocurrent from the dark current.

\section{Experimental section}

\subsection{Cell culture}

Chlamydomonas reinhardtii $\Delta$ petA mutants correspond to cells lacking the cytochrome $\mathrm{b}_{6} \mathrm{f}$ complex (see the photosynthetic chain displayed in Fig. 1). ${ }^{56}$ The cytochrome $b_{6} f$ complex is a quinol:plastocyanin oxidoreductase. Its absence prevents the reoxidation of the plastoquinol $\mathrm{PQH}_{2}$ generated by the lightinduced turnovers of PSII, thus leading to the fast arrest of lightdriven electron flow. In the context of quinones used as redox mediators and PSII acceptors, this cell model allows one to assess their ability to drive the electron flow downstream of Photosystem II and monitor it by fluorescence measurements. ${ }^{37}$ Cells were grown in Tris Acetate Phosphate medium (TAP): Tris base $\left(20 \mathrm{mmol} \mathrm{L}^{-1}\right), \mathrm{NH}_{4} \mathrm{Cl}\left(7 \mathrm{mmol} \mathrm{L}^{-1}\right), \mathrm{MgSO}_{4}\left(0.83 \mathrm{mmol} \mathrm{L}^{-1}\right)$, $\mathrm{CaCl}_{2}\left(0.45 \mathrm{mmol} \mathrm{L}^{-1}\right), \mathrm{K}_{2} \mathrm{HPO}_{4}\left(1.65 \mathrm{mmol} \mathrm{L}{ }^{-1}\right)$, and $\mathrm{KH}_{2} \mathrm{PO}_{4}$ $\left(1.05 \mathrm{mmol} \mathrm{L}^{-1}\right)$ at $25{ }^{\circ} \mathrm{C}$ under rather dim light conditions $\left(50 \mu \mathrm{E} \mathrm{m}^{-2} \mathrm{~s}^{-1}\right)$ to a final concentration of $10^{7}$ cells per $\mathrm{mL}$.

\subsection{Photocurrent measurements}

3.2.1. Red irradiation. Usual electrochemical experiments were performed under red irradiation (LED; $640 \mathrm{~nm}$ ) at an incident light flow equal to $340 \mu \mathrm{E} \mathrm{m}^{-2} \mathrm{~s}^{-1}$ (JTS spectrophotometer, Biologic). After reaching a cell suspension of $10^{7}$ cells per $\mathrm{mL}$ from the culture, cells were centrifuged at $4000 \mathrm{rpm}$ and re-suspended into $16 \mathrm{~mL}$ of TAP supplemented with $7.4 \%$ of phosphate buffered saline (PBS) medium (ref. 18912, Life Technologies; NaCl $140 \mathrm{mmol} \mathrm{L}^{-1}, \mathrm{KCl} 2.68 \mathrm{mmol} \mathrm{L}^{-1}, \mathrm{NaHPO}_{4}$ and $\left.\mathrm{NaH}_{2} \mathrm{PO}_{4} 10 \mathrm{mmol} \mathrm{L}^{-1}\right)$ to reach an adapted ionic strength $\left(\sim 150 \mathrm{mmol} \mathrm{L}^{-1}\right)$ for electrochemical experiments and a final concentration of $10^{7}$ cells per mL. The spectroelectrochemical cuvette is described in ref. 35 and involves a carbon gauze (geometric area $=1 \mathrm{~cm}^{2}$ ) as a working electrode polarized at $E_{\text {applied }}=+650 \mathrm{mV} v s$. Ag/AgCl. Under dark conditions, exogenous quinones (2,6-dichlorobenzoquinone, 2,6-DCBQ or paraphenylbenzoquinone, $\mathrm{PPBQ}$ ) are added at a final concentration equal to $100 \mu \mathrm{mol} \mathrm{L}{ }^{-1}$. When the stability of photocurrents is addressed, light illumination is applied for $30 \mathrm{~min}$ and this leads to a current increase, i.e. the photocurrent. A second pulse (with the same illumination) can be applied once the previous photocurrent returns to the baseline after turning off the illumination. Baseline has been removed as described in ref. 35. All chemicals were purchased from Sigma Aldrich and used without any further purification.

3.2.2. Green irradiation. In some cases, green irradiation (LED; $530 \mathrm{~nm} ; 30 \mu \mathrm{E} \mathrm{m}^{-2} \mathrm{~s}^{-1}$ ) was used. Photocurrents were recorded as described above (notably with a quinone concentration of $100 \mu \mathrm{mol} \mathrm{L}^{-1}$ ). Of note, under green light conditions no photoinactivation occurred. The photocurrent stability was assessed by means of 3 subsequent light pulses after applying the initial pulse and the subsequent return to the baseline. The baseline has been removed as described in ref. 35 .

\subsection{Fluorescence measurements}

All fluorescence measurements have been performed as previously described in ref. 37 and 38 (actinic light: $I^{\circ}=$ $340 \mu \mathrm{E} \mathrm{m}^{-2} \mathrm{~s}^{-1}$; supersaturating pulse $=5000 \mu \mathrm{E} \mathrm{m}^{-2} \mathrm{~s}^{-1}$ ). The actinic light induces the PSII excitation. The supersaturating pulse of excitation light has a much higher intensity than that of the continuous illumination beam. Therefore, the rate at which light is absorbed by PSII largely exceeds that in the subsequent steps downstream from PSII, thus 
promoting the full reduction of the quinone primary electroacceptor $\mathrm{Q}_{\mathrm{A}}$ to $\mathrm{Q}_{\mathrm{A}}{ }^{-}$(closed centers). Because the cell model lacks the $b_{6} f$ complex and the supersaturating pulse is long enough to fully reduce all electron acceptors $(250 \mathrm{~ms})$, no further electron transfer can occur. Each experiment was repeated three times.

Incubation periods were achieved under dark conditions and gentle stirring in order to avoid aggregation either in the presence of quinones (2,6-DCBQ, PPBQ, 2,5-DCBQ or 2,6-DMBQ, see Scheme 1) or in the absence of quinones as a control experiment. Fluorescence measurements were performed every hour for each sample $(V=2 \mathrm{~mL})$.

\section{Conclusions}

In conclusion, we demonstrated through fluorescence and electrochemical measurements that during photosynthetic electron harvesting from a unicellular algae suspension with quinones, a decrease in terms of performance took place because of three different phenomena. The first one is related to the light conditions, i.e. to possible photoinactivation. The other two are side-effects due to the added quinones as redox mediators. On the one hand, a kinetic quenching is observed for all the quinones, especially for PPBQ. On the other hand, an alteration of the ratio of open centers is also observed for chloroquinones that may lead to the decrease of the photochemical rate and a corresponding decrease of recorded photocurrents. Controlling the experimental conditions (nature of the quinone, incubation time, and excitation light) discriminate the different kinds of degradation (from quinones or from light). Therefore, a compromise needs to be found at long timescales between the ability of the quinone to harvest electrons and its poisoning effect. As an example, poor PSII acceptor quinones like DMBQ will not induce any alteration but will require appropriate mutations of the photosynthetic organism to increase its ability to extract electrons from the exogenous quinone acceptor. ${ }^{36}$

To the best of our knowledge, investigations of quinone effects on these biophotoelectrochemical cells are rather scarce. While the different mechanisms of action still remain to be investigated in detail, the results reported here pave the way for future optimizations of quinone use for harvesting bioelectricity from photosynthetic organisms.

\section{Conflicts of interest}

There are no conflicts to declare.

\section{Acknowledgements}

This work has been supported in part by CNRS (UMR8640 and UMR7141), Ecole Normale Supérieure, French Ministry of Research, Faculté des Sciences et Ingénierie - Sorbonne Université, the "Fondation Pierre-Gilles de Gennes pour la Recherche" FPGG0049 and the "Initiative d'Excellence" program from the French State (Grant "DYNAMO", ANR-11LABX-0011-01). G. L. and M. G.-C. are thankful for the Institut
Universitaire de France Fellowship Program. Dr Alison Tebo is gratefully acknowledged for helpful comments.

\section{Notes and references}

1 N. S. Lewis and D. G. Nocera, Proc. Natl. Acad. Sci. U. S. A., 2006, 103, 15729-15735.

2 R. E. Blankenship, D. M. Tiede, J. Barber, G. W. Brudvig, G. Fleming, M. Ghirardi, M. R. Gunner, W. Junge, D. M. Kramer, A. Melis, T. A. Moore, C. C. Moser, D. G. Nocera, A. J. Nozik, D. R. Ort, W. W. Parson, R. C. Prince and R. T. Sayre, Science, 2011, 332, 805-809.

3 X. G. Zhu, S. P. Long and D. R. Ort, in Annu. Rev. Plant Biol., ed. S. Merchant, W. R. Briggs and D. Ort, 2010, vol. 61, pp. 235-261.

4 J. Barber, Chem. Soc. Rev., 2009, 38, 185-196.

5 E. S. Andreiadis, M. Chavarot-Kerlidou, M. Fontecave and V. Artero, Photochem. Photobiol., 2011, 87, 946-964.

6 J. Qi, W. Zhang and R. Cao, Adv. Energy Mater., 2018, 8, 1701620.

7 A. Badura, D. Guschin, B. Esper, T. Kothe, S. Neugebauer, W. Schuhmann and M. Rogner, Electroanalysis, 2008, 20, 1043-1047.

8 S. C. Feifel, K. R. Stieger, H. Lokstein, H. Lux and F. Lisdat, J. Mater. Chem. A, 2015, 3, 12188-12196.

9 V. Hartmann, T. Kothe, S. Poller, E. El-Mohsnawy, M. M. Nowaczyk, N. Plumere, W. Schuhmann and M. Rogner, Phys. Chem. Chem. Phys., 2014, 16, 11936-11941. 10 D. Mersch, C. Y. Lee, J. Z. Zhang, K. Brinkert, J. C. FontecillaCamps, A. W. Rutherford and E. Reisner, J. Am. Chem. Soc., 2015, 137, 8541-8549.

11 O. Yehezkeli, R. Tel-Vered, J. Wasserman, A. Trifonov, D. Michaeli, R. Nechushtai and I. Willner, Nat. Commun., 2012, 3.

12 T. Kothe, S. Poeller, F. Zhao, P. Fortgang, M. Roegner, W. Schuhmann and N. Plumere, Chem.-Eur. J., 2014, 20, 11029-11034.

13 K. P. Sokol, D. Mersch, V. Hartmann, J. Z. Zhang, M. M. Nowaczyk, M. Rogner, A. Ruff, W. Schuhmann, N. Plumere and E. Reisner, Energy Environ. Sci., 2016, 9, 3698-3709.

14 K. Hasan, Y. Dilgin, S. C. Emek, M. Tavahodi, H.-E. Akerlund, P.-A. Albertsson and L. Gorton, ChemElectroChem, 2014, 1, 131-139.

15 J. O. Calkins, Y. Umasankar, H. O'Neill and R. P. Ramasamy, Energy Environ. Sci., 2013, 6, 1891-1900.

16 H. Hamidi, K. Hasan, S. C. Emek, Y. Dilgin, H.-E. Akerlund, P.-A. Albertsson, D. Leech and L. Gorton, ChemSusChem, 2015, 8, 990-993.

17 S. Larom, D. Kallmann, G. Saper, R. Pinhassi, A. Rothschild, H. Dotan, G. Ankonina, G. Schuster and N. Adir, Photosynth. Res., 2015, 126, 161-169.

18 G. Pankratova, D. Pankratov, K. Hasan, H. E. Akerlund, P. A. Albertsson, D. Leech, S. Shleev and L. Gorton, Adv. Energy Mater., 2017, 7.

19 R. I. Pinhassi, D. Kallmann, G. Soper, S. Larom, A. Linkov, A. Boulouis, M. A. Schottler, R. Bock, A. Rothschild, N. Adir and G. Schuster, PLoS One, 2015, 10. 
20 M. Rasmussen, A. Shrier and S. D. Minteer, Phys. Chem. Chem. Phys., 2013, 15, 9062-9065.

21 K. Hasan, R. D. Milton, M. Grattieri, T. Wang, M. Stephanz and S. D. Minteer, ACS Catal., 2017, 7, 2257-2265.

22 K. Hasan, K. V. R. Reddy, V. Essmann, K. Gorecki, P. O. Conghaile, W. Schuhmann, D. Leech, C. Hagerhall and L. Gorton, Electroanalysis, 2015, 27, 118-127.

23 K. Hasan, H. B. Yildiz, E. Sperling, P. O. Conghaile, M. A. Packer, D. Leech, C. Hagerhall and L. Gorton, Phys. Chem. Chem. Phys., 2014, 16, 24676-24680.

24 K. Hasan, E. Cevik, E. Sperling, M. A. Packer, D. Leech and L. Gorton, Adv. Energy Mater., 2015, 5, 1501100.

25 L. Darus, T. Sadakane, P. Ledezma, S. Tsujimura, I. Osadebe, D. Leech, L. Gorton and S. Freguia, J. Electrochem. Soc., 2017, 164, H3037-H3040.

26 K. Hasan, V. Grippo, E. Sperling, M. A. Packer, D. Leech and L. Gorton, ChemElectroChem, 2017, 4, 412-417.

27 N. Sekar, Y. Umasankar and R. P. Ramasamy, Phys. Chem. Chem. Phys., 2014, 16, 7862-7871.

28 A. Anderson, A. Laohavisit, I. K. Blaby, P. Bombelli, C. J. Howe, S. S. Merchant, J. M. Davies and A. G. Smith, Plant Biotechnol. J., 2016, 14, 22-28.

29 P. Bombelli, R. W. Bradley, A. M. Scott, A. J. Philips, A. J. McCormick, S. M. Cruz, A. Anderson, K. Yunus, D. S. Bendall, P. J. Cameron, J. M. Davies, A. G. Smith, C. J. Howe and A. C. Fisher, Energy Environ. Sci., 2011, 4, 4690-4698.

30 R. W. Bradley, P. Bombelli, D. J. Lea-Smith and C. J. Howe, Phys. Chem. Chem. Phys., 2013, 15, 13611-13618.

31 B. De Caprariis, P. De Filippis, A. Di Battista, L. Di Palma and M. Scarsella, in Ibic2014: 4th International Conference on Industrial Biotechnology, ed. E. Bardone, M. Bravi and T. Keshavarz, 2014, vol. 38, pp. 523-528.

32 L. F. Huang, J. Y. Lin, K. Y. Pan, C. K. Huang and Y. K. Chu, Int. J. Mol. Sci., 2015, 16, 19308-19325.

33 X. M. Li, T. X. Liu, K. Wang and T. D. Waite, Environ. Sci. Technol., 2015, 49, 1392-1399.

34 Z. A. Chen, Y. X. Lyu, K. Y. Wang, X. L. Dong, M. C. Deng, C. M. Bai, Y. P. Xu, W. Zhang and Z. M. Liu, Int. J. Hydrogen Energy, 2013, 38, 13045-13049.

35 G. Longatte, F. Rappaport, F. A. Wollman, M. Guille-Collignon and F. Lemaitre, Electrochim. Acta, 2017, 236, 327-332.

36 H. Y. Fu, D. Picot, Y. Choquet, G. Longatte, A. Sayegh, J. Delacotte, M. Guille-Collignon, F. Lemaitre, F. Rappaport and F. A. Wollman, Nat. Commun., 2017, 8, 15274.

37 G. Longatte, H. Y. Fu, O. Buriez, E. Labbe, F. A. Wollman, C. Amatore, F. Rappaport, M. Guille-Collignon and F. Lemaitre, Biophys. Chem., 2015, 205, 1-8.

38 G. Longatte, F. Rappaport, F. A. Wollman, M. GuilleCollignon and F. Lemaitre, Photochem. Photobiol. Sci., 2016, 15, 969-979.

39 G. Longatte, M. Guille-Collignon and F. Lemaitre, ChemPhysChem, 2017, 18, 2643-2650.

40 S. Izawa, in Methods Enzymol., ed. A. San Pietro, 1980, pp. P413-434.

41 A continuous illumination over several hours leads to a progressive decrease of the photocurrent until reaching the baseline level (see ref. 35). This way of estimating the stability of the system was not considered here because it cannot help to distinguish light-dependent and lightindependent phenomena.

42 T. Roach and A. Krieger-Liszkay, Curr. Protein Pept. Sci., 2014, 15, 351-362.

43 J. L. Bolton and T. Dunlap, Chem. Res. Toxicol., 2017, 30, 1337.

44 J. L. Bolton, M. A. Trush, T. M. Penning, G. Dryhurst and T. J. Monks, Chem. Res. Toxicol., 2000, 13, 135-160.

45 R. Delosme, P. Joliot and J. Lavorel, C. R. Hebd. Seances Acad. Sci., 1959, 249, 1409-1411.

46 B. Genty, J. M. Briantais and N. R. Baker, Biochim. Biophys. Acta, 1989, 990, 87-92.

47 K. Maxwell and G. N. Johnson, J. Exp. Bot., 2000, 51, 659-668. 48 K. K. Karukstis, S. C. Boegeman, J. A. Fruetel, S. M. Gruber and M. H. Terris, Biochim. Biophys. Acta, 1987, 891, 256-264.

49 K. K. Karukstis, S. M. Gruber, J. A. Fruetel and S. C. Boegeman, Biochim. Biophys. Acta, 1988, 932, 84-90.

50 S. Hay, B. B. Wallace, T. A. Smith, K. P. Ghiggino and T. Wydrzynski, Proc. Natl. Acad. Sci. U. S. A., 2004, 101, 17675-17680.

51 V. Petrouleas and B. A. Diner, Biochim. Biophys. Acta, 1987, 893, 126-137.

52 C. Preston and C. Critchley, Photosynth. Res., 1988, 16, 187202.

53 X. W. Guo and H. Mayr, J. Am. Chem. Soc., 2014, 136, 1149911512.

54 Y. Song and G. R. Buettner, Free Radical Biol. Med., 2010, 49, 919-962.

55 The photocurrent recorded with 2,6-DCBQ is almost double the one with PPBQ. Indeed, the different conditions (high incubation time for PPBQ) make comparison difficult. This may result from several reasons. For instance, the high incubation time with PPBQ allows the kinetic quenching to take place and leads to a lower photocurrent. Nevertheless, other explanations are not related to the side-effects of the quinones. This rather suggests that 2,6DCBQ is intrinsically more able to harvest electrons from PSII than PPBQ. This trend was already observed in previous fluorescence measurements (see ref. 37) where the percentage of open centers $\Phi$ (i.e. the extraction yield) was displayed as a function of the quinone concentration. The initial slope $\rho_{0}$ and the maximum value of $\Phi$ were shown to be higher for 2,6-DCBQ $\left(4.1 \times 10^{-2} \mu \mathrm{mol}^{-1} \mathrm{~L}\right.$ and 0.58$)$ than for PPBQ $\left(1.7 \times 10^{-2} \mu \mathrm{mol}^{-1} \mathrm{~L}\right.$ and 0.48 , respectively). While the redox potentials $\left(\mathrm{Q} / \mathrm{QH}_{2}\right.$ couple) are rather similar, they show a good correlation with the maximum value of $\Phi$ (when the electron transfer controls the extraction) according to Marcus theory (see ref. 38). Furthermore, the differences in terms of the initial slope $\rho_{0}$ are related to conditions where the mass transfer limits the harvesting. In our case, this probably results from a higher affinity of the dichloroquinone with the $\mathrm{Q}_{\mathrm{B}}$ pocket.

56 B. Rimbault, D. Esposito, D. Drapier, Y. Choquet, D. Stern and F. A. Wollman, Mol. Gen. Genet., 2000, 264, 486-491. 